BMJ Nutrition,

Prevention \& Health

\section{Impact of smoking on COVID-19 outcomes: a HOPE Registry subanalysis}

To cite: Espejo-Paeres C, Núñez-Gil IJ, Estrada V, et al. Impact of smoking on COVID-19 outcomes: a HOPE Registry subanalysis. BMJ Nutrition, Prevention \& Health 2021;4:e00269. doi:10.1136/

\section{- Additional supplemental} material is published online only. To view, please visit the journal online (http://dx.doi.org/10. 1136/bmjnph-2021-000269).

For numbered affiliations see end of article.

Correspondence to Dr Carolina Espejo-Paeres, Cardiology, Hospital Clinico Universitario San Carlos Instituto Cardiovascular, Madrid 28040, Spain;

carolina.espejo.paeres@gmail. com

CE-P and IJN-G contributed equally. Accepted 23 May 2021 Published Online First 17 June 2021 bmjnph-2021-000269

Received 3 March 2021

\section{ABSTRACT}

Background Smoking has been associated with poorer outcomes in relation to COVID-19. Smokers have higher risk of mortality and have a more severe clinical course. There is paucity of data available on this issue, and a definitive link between smoking and COVID-19 prognosis has yet to be established.

Methods We included 5224 patients with COVID-19 with an available smoking history in a multicentre international registry Health Outcome Predictive Evaluation for COVID-19 (NCT04334291). Patients were included following an inhospital admission with a COVID-19 diagnosis. We analysed the outcomes of patients with a current or prior history of smoking compared with the non-smoking group. The primary endpoint was all-cause in-hospital death.

Results Finally, 5224 patients with COVID-19 with available smoking status were analysed. A total of 3983 (67.9\%) patients were non-smokers, 934 (15.9\%) were former smokers and 307 (5.2\%) were active smokers.

The median age was 66 years (IQR 52.0-77.0) and $58.6 \%$ were male. The most frequent comorbidities were hypertension (48.5\%) and dyslipidaemia (33.0\%). A relevant lung disease was present in 19.4\%. In-hospital complications such sepsis (23.6\%) and embolic events $(4.3 \%)$ occurred more frequently in the smoker group $(p<0.001$ for both). All cause-death was higher among smokers (active or former smokers) compared with non-smokers ( 27.6 vs $18.4 \%, \mathrm{p}<0.001)$. Following a multivariate analysis, current smoking was considered as an independent predictor of mortality (OR 1.77, 95\% Cl 1.11 to $2.82, p=0.017$ ) and a combined endpoint of severe disease (OR 1.68, 95\% $\mathrm{Cl} 1.16$ to $2.43, \mathrm{p}=0.006$ ).

Conclusion Smoking has a negative prognostic impact on patients hospitalised with COVID-19.

Check for updates

(C) Author(s) (or their employer(s)) 2021. Re-use permitted under CC BY-NC. No commercial re-use. See rights and permissions. Published by BMJ.

\section{INTRODUCTION}

COVID-19 was declared a pandemic on 11 March 2020 by the WHO. ${ }^{1}$ COVID-2019 has a wide spectrum of manifestations ranging from subclinical infection to acute respiratory distress syndrome (ARDS) and multiorgan

\section{What this paper adds}

- It has been described that a history of smoking is associated with higher rates of mortality and complications in patients with COVID-19. Contrastingly, it has been suggested that tobacco may have a 'protective' role in COVID-19 infections.

- The strengths of this study are the large sample size and adjusting for confusion factors to evaluate the independent role of tobacco in the course of SARSCoV-2 infections. We included former smokers as well in our analysis since both current and former smokers share characteristics and underlying respiratory comorbidities.

- Main limitations of this study are that some variables such as packs-year of smoking or smoke-free years were not recorded, as well as the bias given the observational nature of the study.

failure. $^{23}$ Although some poor prognostic factors have been observed, ${ }^{4}$ its clinical course remains unpredictable.

Since pulmonary alveolar epithelial cells are one of the targets of SARS-CoV-2, underlying respiratory risk factors may play a role in modifying respiratory response. Available findings regarding smokers are inconsistent, and the impact of smoking on SARS-CoV-2 infection is uncertain. ${ }^{4-7}$

A large case series from China reported a higher prevalence of active smokers in severe COVID-19 infections, in comparison to milder cases. ${ }^{4}$ Nevertheless, some studies failed to demonstrate a link between smoking and poorer prognosis of the disease. Even some previous series suggested a theoretical 'protective' effect of smoking habit. ${ }^{78}$ This last hypothesis can be deduced from the lower rates of smoking observed in patients 


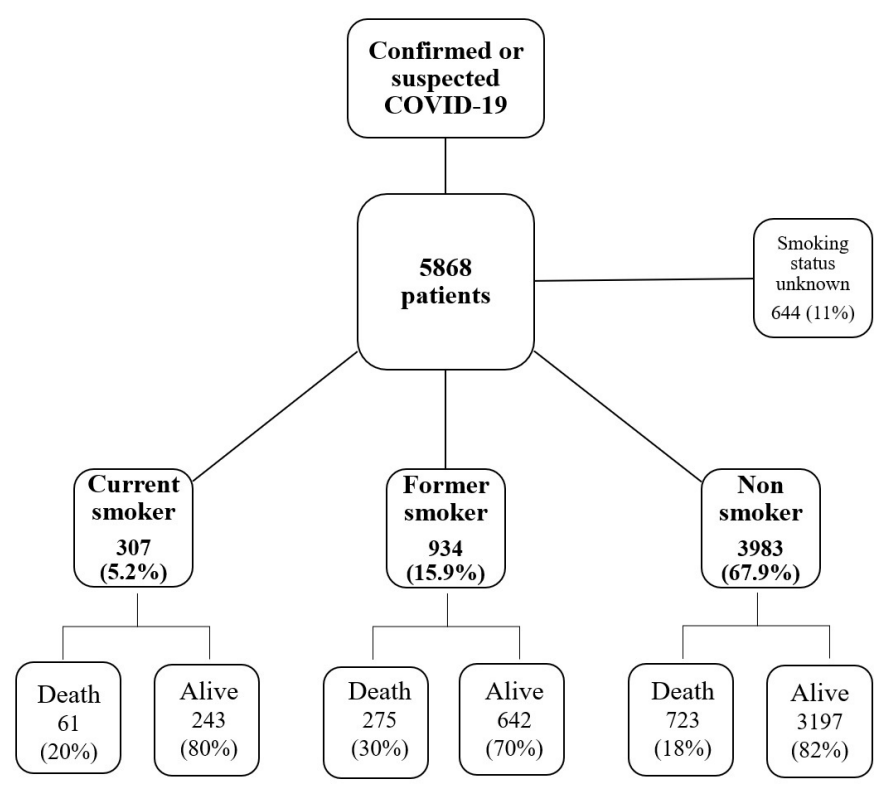

Figure 1 Study flow diagram.

with COVID-19 in comparison with the general population. The prevalence of smokers among SARS-CoV-2infected patients has been estimated between $1.4 \%$ and $12.5 \%$ according to different studies. ${ }^{5-15}$ These rates are notably lower than those recorded in the Chinese general population $(25.2 \%) .^{16}$

The pathophysiology of lung damage has not been fully understood. It has been suggested that high levels of proinflammatory cytokines in serum can induce the hyperinnate inflammatory response. This cascade produces a 'cytokine release syndrome' with an overproduction of immune cells and cytokines, which leads to an ARDS and septic shock. ${ }^{17-20}$ Smoking may modulate the immune response and smokers could present an attenuated immune response presenting lower levels of inflammation markers compared with nonsmokers. $^{21} 22$

On the one hand, the ACE2 protein is known to play a role in the infection's mechanism. The ACE2 protein is expressed on the surface of lung type 2 pneumocytes and is the principal receptor molecule for SARS-CoV-2. ${ }^{23-25}$ On the other hand, some authors have described decreased levels of ACE2 in smokers. ${ }^{26} 27$ Conversely, it has been suggested that ACE2 is upregulated on the airway epithelium of smokers. In a study in resected lung specimens, Leung and coworkers found an increase of ACE2 gene expression in patients with chronic obstructive pulmonary disease (COPD). Likewise, a higher ACE2 gene expression was observed in smokers when compared with non-smoker individuals. ${ }^{28} 29$ The question of whether smokers are more prone to contract SARS-CoV-2 infection remains unresolved.

Based on the aforementioned, we aimed to assess if smokers are more likely to die or develop more severe forms of COVID-19.

\section{METHODS}

\section{Study design and population}

We conducted a cohort study of 5868 consecutive patients who were hospitalised with confirmed or highly suspected COVID-19 infection. Smoking history was available in the 5224 patients included in the final analysis (figure 1). The patients were entered in the Health Outcome Predictive Evaluation for COVID-19 Registry (NCT04334291), a multicentre international registry without conflicts of interest, designed as an ambispective cohort. In this multicentre registry, we included the data from 40 centres from seven countries. From 23 March 2020 to 5 May 2020, patients discharged from the hospital (deceased or alive) with COVID-19 diagnosis were included in the registry. Epidemiological and clinical data were obtained from electronic medical records, and the data were stored in an anonymised fashion.

\section{Definitions and study endpoints}

We assessed the impact of smoking on the prognosis of 5224 patients with COVID-19. We defined three study groups according to smoking status. Patients were classified in active smokers, former smokers and non- smokers. We evaluated the differences in baseline characteristics, clinical presentation and treatment, according to these groups. Likewise, we performed an age-stratified analysis of mortality and complications in each smoking group. Age groups were divided into quartiles as follows: $(1)<52$ years old, (2) 52-66 years old, (3) 66-77 years old and (4) $>77$ years old.

The primary endpoint was defined as all-cause in-hospital death. A combined secondary endpoint was established as a composite of intensive care unit (ICU) admission, need of prone position or death. Other secondary outcomes assessed included in-hospital complications such as ICU admission, respiratory insufficiency, pneumonia, sepsis, systemic inflammatory response syndrome (SIRS) and embolic events.

\section{Patient and public involvement}

The research question and outcome measures were informed according to experience. Due to the pandemic situation, it was not appropriate to involve patients or the public in the design of our research and recruitment to or conduct of the study. Results may be disseminated on reasonable request.

\section{Statistical analysis}

The data are presented as mean (SD) for continuous variables with a normal distribution, median (IQR) for continuous variables with a non-normal distribution and as frequency (\%) for categorical variables. Student's t-test and the Mann-Whitney $\mathrm{U}$ test were used to compare continuous variables with normal and non-normal distributions, when needed. The $\chi^{2}$ test or Fisher's exact test was used to compare categorical variables. Univariate analysis was performed for qualitative variables and reported as ORs with $95 \%$ CIs. Given the multiplicity of variables, only 
factors with a $p$ value of $<0.01$ on the mentioned univariate analysis in the smoker cohort were entered into the multivariate analysis (binary logistic regression) to define independent risk factors for the principal outcome and focusing on the smoking status (current, former or never). Mortality analysis was performed using KaplanMeier estimates and log-rank tests to compare factors. Two-sided $p$ values of $<0.05$ were accepted as statistically significant. Likewise, in order to eliminate potential confounding factors, propensity scores for mortality and the combined endpoint were performed. Statistical analysis was performed using SPSS V. 22.0 and STATA V. 14.0.

\section{RESULTS}

A total of 5224 patients with COVID-19 were included in this analysis. The majority patients, 3983 (67.9\%), were non-smokers, while $934(15.9 \%)$ were former smokers and only 307 (5.2\%) were active smokers. Smoking habits were not available in 644 patients $(11 \%)$; thus, finally, 5224 patients were entered in the study (figure 1). The median age was 66 years (IQR 52.0-77.0) and $3060(58.6 \%)$ were male. Most individuals were Caucasian (4333, 82.9\%) followed by Hispanic ethnicity $(710,13.6 \%)$.

In the overall cohort, the most frequent comorbidities were hypertension $(2626,48.5 \%)$ and dyslipidaemia $(1716,33.0 \%)$. Other conditions included heart disease (of any form; 1191, 23\%) and obesity $(981,22.1 \%$ ). A relevant lung disease was present in 1012 patients (19.4\%). The most frequent lung disease was COPD (39.4\%) followed by asthma (26.9\%).

Comparing smoking patterns, we found that former smokers were older and had a higher comorbidity burden compared with both active and non-smoker groups. Ex-smokers had higher rates of hypertension (64.3\%), dyslipidaemia $(48.6 \%)$ and obesity $(30.6 \%)$, as well as a higher prevalence of lung disease $(39.3 \%)$ and heart disease $(38.3 \%) \quad(\mathrm{p}<0.001$ for all $)$. Ex-smokers presented the highest prevalence of COPD (24.6\%) compared with smokers $(16.3 \%)$ and non-smokers (3\%) $(\mathrm{p}<0.001)$. Asthma was predominantly observed among non-smokers (5.7\%). Consequently, cardiovascular medications such as ACE inhibitor/angiotensin receptor blockers, antiplatelets or inhaled beta agonist were more prevalent among former smokers. Differences in baseline characteristics and previous treatment are displayed in table 1 .

Regarding clinical manifestations, current smokers presented with different symptoms complaining of anosmia, dysgeusia and sore throat to a higher degree than the two other groups. The most common symptoms were fever $(73 \%)$ and cough $(65.6 \%)$, whereas dyspnoea was less frequently described among smokers. It is worth noting that acute-phase reactants such as C reactive protein, lactate dehydrogenase and ferritin were less frequently elevated in the smoking group, while white cell count was higher than in non-smokers or ex-smokers. Clinical presentation and analytical results are described in online supplemental table S1.
The current smoker group received more beta interferon but less antibiotics or prophylactic anticoagulation compared with both non-smoker and ex-smoker groups $(\mathrm{p}<0.001)$ (online supplemental table S2). In line with this, in-hospital complications such as sepsis $(23.6 \%)$ and embolic events $(4.3 \%)$ occurred more frequently in the smoker group ( $\mathrm{p}<0.001$ for both) (online supplemental table S3).

The secondary endpoint of ICU admission was greater among the active-smoker group in comparison with the former-smoker group and the non-smoker patients $(\mathrm{p}<0.001)$, while the prone position was more frequently used among ex-smokers (online supplemental table S3).

In the univariate analysis, all-cause death was higher in smokers $(20.1 \%)$ when compared with non-smokers $(18.4 \%) \quad(\mathrm{p}<0.001)$, while the highest mortality was observed among the former-smoker group (30.0\%) $(\mathrm{p}<0.001)$. Mortality according to age groups is shown in figure 2 and online supplemental table S4.

The impact of a smoking history was then compared with the absence of a smoking history. All-cause in-hospital mortality and the combined endpoint (ICU admission, prone, death) are depicted in online supplemental table S5.

Following this, a multivariate analysis was performed. After adjusting for confounding factors, current smokers presented a greater risk of death from all causes (OR 1.77, 95\% CI 1.11 to 2.82, $\mathrm{p}=0.017$ ) when compared with nonsmokers. Likewise, former smokers had an increased risk of death compared with non-smokers (OR 1.32 95\% CI 1.0 to $1.73 \mathrm{p}=0.049$ ), but this independent risk was not as strong as that observed in the current smokers' group.

Other independent predictors of mortality were older age, hypertension, previous heart disease and elevated LDH. Moreover, we performed a multivariate analysis for a combined endpoint of death, ICU admission or need of prone position. As well, the highest risk for the combined endpoint was observed among active smokers when compared with non-smokers (OR 1.68, 95\% CI 1.16 to $2.43, \mathrm{p}=0.006$ ). There were no significant differences in the combined endpoint between former smokers and non-smokers after adjusting for comorbidities (OR 1.09, $95 \%$ CI 0.86 to $1.39, \mathrm{p}=0.467)$. The multivariate analysis is presented in the table 2.

In online supplemental table $\mathrm{S} 6$ propensity scores for mortality and the combined endpoint are depicted. In propensity scores, any kind of smokers (former or current) were compared with non-smokers.

Kaplan-Meier survival curve for all-cause mortality is displayed in figure 3 .

\section{DISCUSSION}

The main finding in our study was the fact that current smoking was independently associated with a twofold increased risk of mortality compared with non-smoking, after adjusting for confounding factors in a large international cohort admitted with COVID-19. 
Table 1 Baseline characteristics and previous treatment

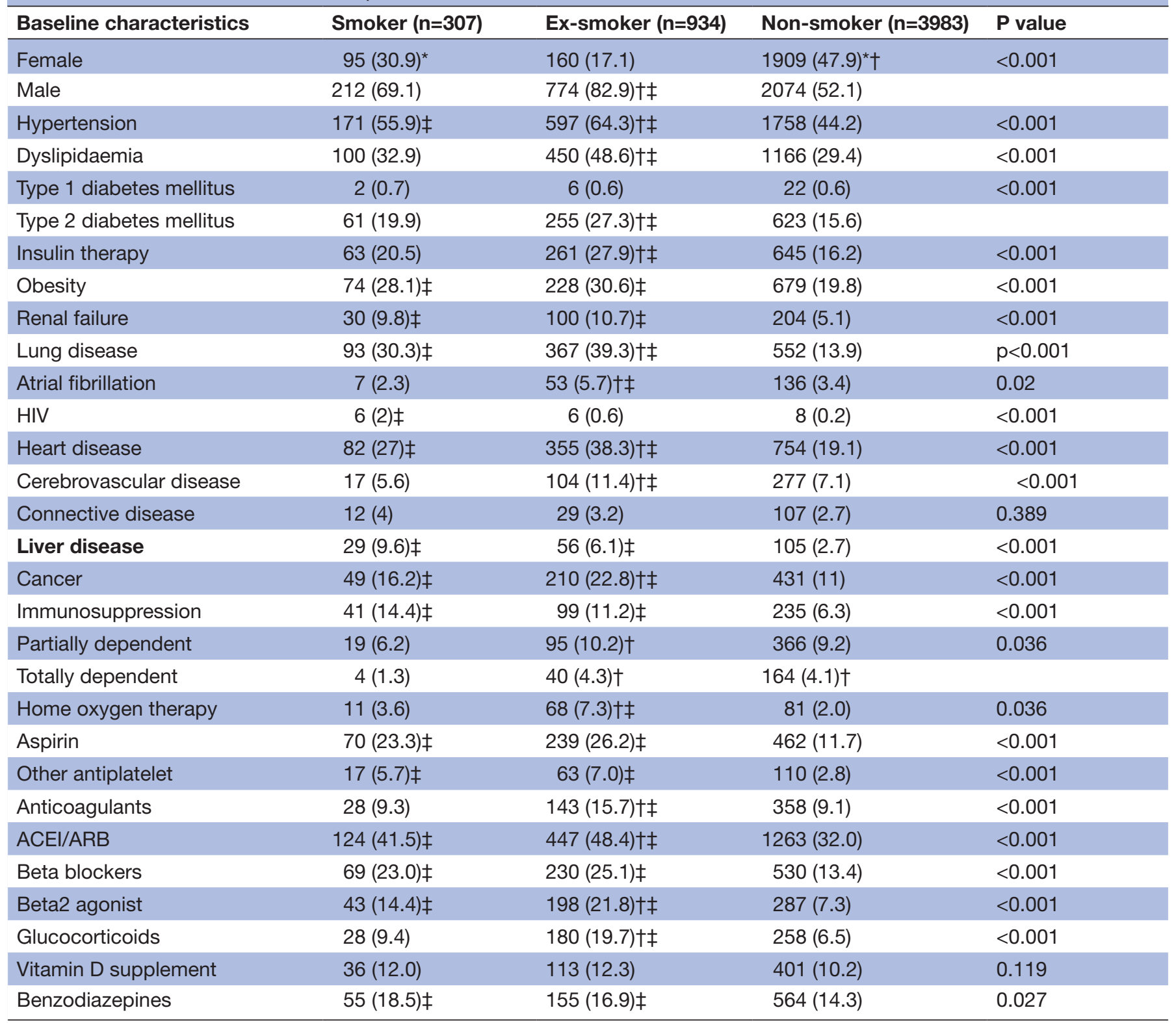

Values are $\mathrm{n}(\%)$. All $\mathrm{p}$ values were determined by using an analysis of variance with Bonferroni method.

${ }^{*} \mathrm{P}<0.05$ compared to ex-smokers.

$\dagger \mathrm{P}<0.05$ compared to smoker subjects.

$\ddagger \mathrm{P}<0.05$ compared to non-smoker subjects.

ACEI, ACE inhibitor; ARB, angiotensin receptor blocker.

As well, active smokers presented a higher risk for critical illness (combined endpoint of death, prone position and ICU admission) in comparison with non-smokers (1.7-fold). Interestingly, in spite of the fact that former smokers were sicker, the risk of mortality was not as strong as the risk of current smokers after adjusting for comorbidities.

According to previous series, smoking has been associated with higher mortality and complication rates in patients with COVID-19. ${ }^{4-7}$ Our aim was to clarify if the detrimental effect of smoking was independently associated with poor prognosis in COVID-19 after adjusting for other factors.
SARS-CoV-2 targets pulmonary alveolar epithelial cells and can cause severe pneumonia and respiratory distress. ${ }^{2-5}$ Thus, underlying respiratory risk factors such as previous lung disease or smoking may alter the respiratory response. Some studies have shown that COPD is associated with a worse prognosis of COVID-19. ${ }^{515}$

The pathogenesis of acute lung injury remains largely unknown. It has been suggested that high levels of proinflammatory cytokines in serum can induce the hyperinnate inflammatory response. The hyperinnate inflammatory response leads to the activation of Th1 cell-mediated immunity and accumulation of alveolar macrophages and neutrophils. This cascade produces a 'cytokine release syndrome' 


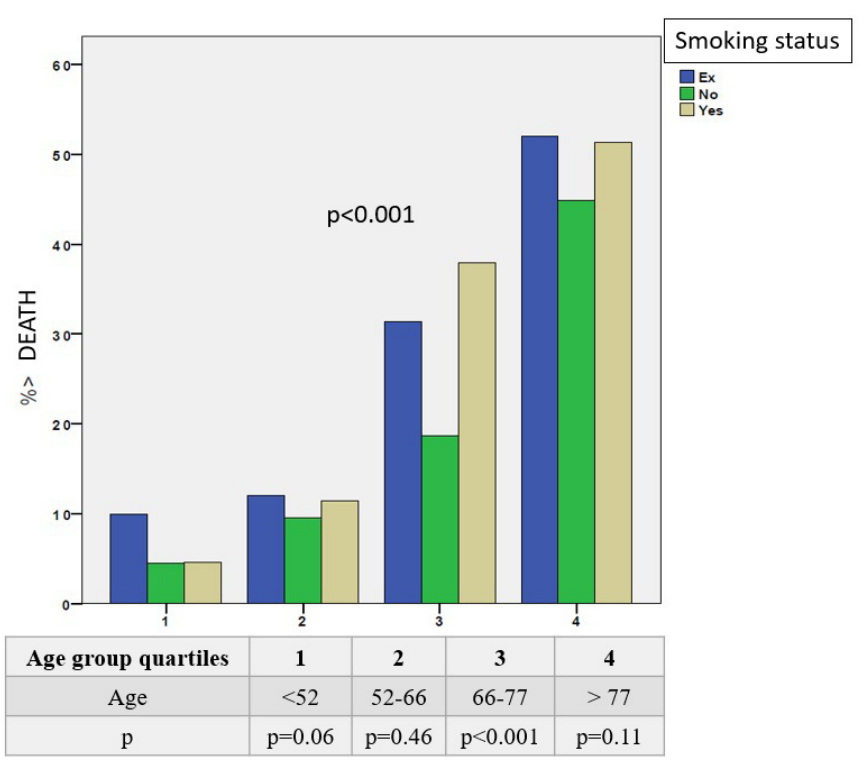

Figure 2 All-cause in-hospital death according to smoking status, stratified by age.

with an overproduction of immune cells and cytokines, which leads to an ARDS and septic shock. ${ }^{17-20}$ Smoking may alter and attenuate immune response by lowering inflammatory marker levels. ${ }^{21}{ }^{22}$ In line with this, smokers in our cohort presented lower levels of $\mathrm{C}$ reactive protein, ferritin and dehydrogenase lactate when inflammatory marker levels were compared between groups (online supplemental table S1). Likewise, SIRS was less frequently observed in the current smoker group (online supplemental table S3).

Despite these findings, globally, smoking has detrimental effects on the immune system and infectious response, and has been associated with a worse prognosis of pulmonary disease. ${ }^{17}{ }^{18}$ Moreover, smokers were noted to have higher mortality in the previous MERS-CoV outbreak compared with non-smokers $(37 \%$ vs $19 \%$, $\mathrm{OR}=3.14,95 \%$ CI 1.10 to $9.24, \mathrm{n}=146){ }^{30}$

Concerning SARS-CoV-2 infection, previous studies have suggested that active smokers and former smokers are more prone to develop severe COVID-19 infections. Despite the apparent logical link between smoking and COVID-19 prognosis, this relationship has not been fully established. In some previous studies, statistical significance was not reached; sample sizes were small; and results were not entirely adjusted for other confounding factors. ${ }^{4-7}$

Reviewing the available previous data, Guan et al described clinical characteristics and outcomes of 1099 patients with COVID-19 from China. This study reported a higher prevalence of active-smokers in severe COVID-19 $(16.9 \%)$ compared with non-severe disease (11.8\%). However, no statistical analysis for evaluating any association was performed. ${ }^{4}$

Moreover, Zhao et al conducted a meta-analysis with 11 case series. They studied the impact of smoking on the severity of COVID-19 among 2002 patients. This study concluded that active smoking increases the risk of severe COVID-19 (fixed effect model, OR=1.98, 95\% CI 1.29 to
Table 2 Multivariate analysis for in-hospital mortality and for secondary combined endpoint

\begin{tabular}{|c|c|c|c|}
\hline & \multicolumn{3}{|c|}{$\begin{array}{l}\text { Multivariate analysis for in-hospita } \\
\text { mortality }\end{array}$} \\
\hline & OR & $95 \% \mathbf{C l}$ & $P$ value \\
\hline Current smoker & 1.77 & 1.11 to 2.82 & 0.017 \\
\hline Former smoker & 1.32 & 1.00 to 1.73 & 0.049 \\
\hline Age 52-66 years old & 1.74 & 1.10 to 2.79 & 0.020 \\
\hline Age 66-77 years old & 4.56 & 2.90 to 7.19 & $<0.001$ \\
\hline Age $>77$ years old & 10.63 & 6.78 to 16.66 & $<0.001$ \\
\hline Hypertension & 1.71 & 1.33 to 2.2 & $<0.001$ \\
\hline Lung disease & 1.06 & 0.81 to 1.39 & 0.679 \\
\hline Any cardiac disease & 1.38 & 1.08 to 1.76 & 0.010 \\
\hline Elevated CRP & 2.11 & 1.23 to 3.63 & 0.007 \\
\hline Elevated LDH & 2.61 & 1.91 to 3.58 & $<0.001$ \\
\hline \multirow[t]{3}{*}{ Elevated ferritin } & 1.22 & 0.96 to 1.53 & 0.101 \\
\hline & \multicolumn{3}{|c|}{$\begin{array}{l}\text { Multivariate analysis for the } \\
\text { composite endpoint }^{\star}\end{array}$} \\
\hline & OR & $95 \% \mathrm{Cl}$ & $P$ value \\
\hline Current smoker & 1.68 & 1.16 to 2.43 & 0.006 \\
\hline Former smoker & 1.09 & 0.86 to 1.39 & 0.467 \\
\hline Age 52-66 years old & 1.33 & 1.01 to 1.77 & 0.044 \\
\hline Age 66-77 years old & 1.77 & 1.31 to 2.40 & $<0.001$ \\
\hline Age $>77$ years old & 2.64 & 1.95 to 3.57 & $<0.001$ \\
\hline Hypertension & 1.65 & 1.35 to 2.03 & $<0.001$ \\
\hline Lung disease & 1.26 & 1.00 to 1.58 & 0.054 \\
\hline Any cardiac disease & 1.53 & 1.23 to 1.91 & $<0.001$ \\
\hline Elevated CRP & 2.27 & 1.50 to 3.44 & $<0.001$ \\
\hline Elevated LDH & 2.16 & 1.70 to 2.75 & $<0.001$ \\
\hline Elevated ferritin & 1.61 & 1.32 to 1.96 & $<0.001$ \\
\hline
\end{tabular}

Statistically significant $p$ value: $p<0.05$.

*Composite endpoint of intensive care unit admission, prone position or death.

$\mathrm{Cl}$, Confidence interval; CRP, C reactive protein; LDH, lactate dehydrogenase.

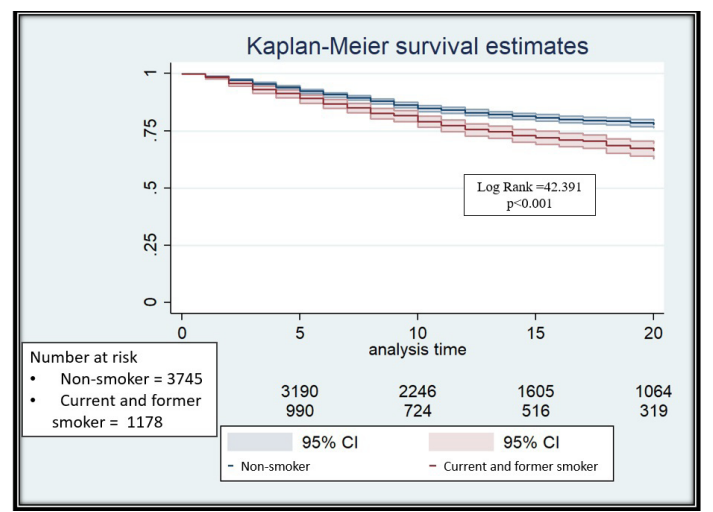

Figure 3 Kaplan-Meier survival curve free from all-cause death, according to smoking status. 
3.05) by around twofold. ${ }^{5}$ Results were heavily influenced by one study ${ }^{4}$ and after removing it from analysis, association was not reached with the OR of 1.55 (95\% CI 0.83 to 2.87$).^{5}$

In a similar fashion, Liu et al studied 78 patients with COVID-19. They found a higher proportion of smokers (27.3\%) among adverse outcome group, compared with the group that showed improvement or stabilisation $(3.0 \%)(\mathrm{p}=0.018)$. In their multivariate logistic regression analysis, the history of smoking was a risk factor of disease progression $(\mathrm{OR}=14.28,95 \%$ CI 1.58 to $25.00, \mathrm{p}=0.018) .{ }^{6}$

Furthermore, in a meta-analysis conducted by Patanavanich and Glantz, a total of 11590 patients with COVID-19 from 19 studies were included. In the overall cohort, $18.4 \%$ of the patients developed disease progression. Afterwards, results between smokers and non-smokers were compared. Smokers presented a higher rate of disease progression $(29.8 \%)$ in contrast with non-smokers $(17.6 \%)$ with a twofold increased risk for smokers (OR $1.91,95 \%$ CI 1.42 to $2.59, p=0.01) .{ }^{31}$

In our cohort, pernicious effects of smoking have been observed by the finding of a relationship between smoking and worse outcomes in patients with COVID-19. Mortality was significantly higher among patients with a smoking history (both former and current) than in the non-smoker group (27.6 vs $18.4 \%, \mathrm{p}<0.001$ ). Likewise, a more severe disease was associated to both present and past smoking history (composite endpoint: 36.2 vs $26.1 \%$, $\mathrm{p}<0.001$; online supplemental table S5).

Our results are in line with those found in a meta-analysis conducted by Jiménez-Ruiz et al. This study analysed data from 34 studies including a total of 6487 patients with SARS-CoV-2 infections. This meta-analysis showed a worse clinical course in current and former smokers (OR 1.96, 95\% CI 1.36 to 2.83 ), as well as a greater risk of critical illness (OR 1.79, 95\% CI 1.19 to 2.70), when compared with non-smokers. ${ }^{32}$

Moreover, Lowe et al evaluated the association between cumulative smoking exposure, as measured by pack-years, with COVID-19 outcomes. They studied a cohort of 7102 patients recovered in Cleveland Clinic who tested positive for COVID-19. Eighty-five per cent (6020) of them were non-smokers; $2.4 \%$ (172) were current smokers; and $12.8 \%$ (910) were former smokers. As well, they compared non-smokers with patients smoking 0-10, 10-30 and more than 30 pack-years, respectively. They found an association between the risk of bad outcomes and the number of pack-years, with a 1.89 and 2.25-fold increased risk of mortality and hospitalisation, respectively, among those patients smoking more than 30 pack-years. This relationship was dose-dependent with a progressive increment of risk according to the number of pack-years. They conclude that smoking is an independent risk factor for hospital admission and mortality in COVID-19. ${ }^{33}$

Likewise, a meta-analysis conducted by Vardavas reviewed five studies on COVID-19. All studies included patients' smoking status with sample sizes ranging from 41 to 1099 patients. In all these studies, there was a higher prevalence of both current and former smokers among more severe cases (patients who needed ICU support, mechanical ventilation or who had died; relative risk (RR): 2.4, IQR 1.43-4.04). ${ }^{34}$

Another retrospective study conducted by Adrish et al analysed 1173 patients with COVID-19 and smoking habit available. Among them, 837 patients never smoked while 336 were either current or past smokers. In this analysis, smokers developed more critical illness requiring mechanical ventilation ( $47 \%$ vs $37 \% \mathrm{p}=0.005$ ). Univariate Cox model for survival showed that only current smokers had higher risk of death compared with never smokers (HR $1.61,95 \%$ CI 1.22 to $2.12, \mathrm{p}<0.001) .{ }^{35}$

In our cohort, to clarify if the impact of smoking on COVID-19 outcome is rather linked to the smokingrelated comorbidities, a multivariate logistic regression analysis was performed. After adjusting by confounding factors, mortality and severity (defined as the composite endpoint) were still higher among smokers compared with never smokers (multivariate analysis in table 2). Indeed, one of the most interesting findings in this work is the fact that active smokers, even if they presented less comorbidities than former smokers, presented the greatest risk of mortality and severity, once confounding factors were adjusted. Current smoking was found to be an independent predictor of mortality and poor prognosis in COVID-19. Former smokers presented a slightly increased risk of mortality (1.3-fold), but there were no statistically significant differences for the combined endpoint in contrast with non-smokers.

In our study, ex-smokers had a greater burden of comorbidities compared with the other two groups. It might explain a higher crude mortality rate among ex-smokers in comparison with active smokers.

Unlike our cohort, other studies did not include ex-smokers in their analysis. ${ }^{9}$ It is worth highlighting the importance of including ex-smokers, since both current and former smokers share characteristics and underlying respiratory comorbidities. Moreover, both groups have a similar expression of ACE2 receptors. Cai observed a higher ACE2 gene expression in the airway epithelia of healthy patients with a current or previous history of smoking compared with non-smokers. ${ }^{28}$

Contrastingly, it has been widely questioned whether a history of smoking contributes to an increased risk of contracting COVID-19. An important mechanism of SARS-COV2 infection relates to the levels of ACE 2 proteins that are produced. While the hyperinnate inflammatory response is mainly related to the clinical course of the disease, this second mechanism may play a role in the susceptibility to infection. The ACE2 protein is expressed on the surface of lung type 2 pneumocytes and is the principal receptor molecule for SARS-CoV-2. Some authors have described decreased levels of ACE2 in smokers,${ }^{2627}$ which proposes a protective role of smoking. This mechanism suggests that in the ACE/ANG II/ AT1R arm, nicotine increases the expression and activity of renin, ACE and AT1R, whereas in the compensatory 
ACE2/ANG-(1-7)/MasR arm, nicotine downregulates the expression and activity of ACE2 and AT2R, thus suggesting a possible contribution of acetylcholine receptors in ACE2 regulation (nicotine) ${ }^{27}$

A theoretical 'protective' role of tobacco in COVID-19 infection has been suggested. It is worth noting the lower rates of smoking observed in patients with COVID-19 in comparison with the general population. This may indicate a lower susceptibility to the infection in individuals with a smoking history.

Previous Chinese studies have shown low rates of current smokers among SARS-CoV-2-infected patients $(1.4 \%-12.5 \%),{ }^{5-15}$ lower than the reported prevalence of smoking in China (25.2\%). ${ }^{16}$ Moreover, possible selection biases need to be considered. It is remarkable the median age of patients ranged from 38 to 59.7 years in the previously mentioned series. ${ }^{6-14}$ These ages are strikingly lower than expected results and differ notably from our cohort with a median age of 66 years (IQR $52.0-77.0)$.

Similarly, Miyara et al studied 482 patients with COVID-19 to evaluate smoker's susceptibility to develop SARS-CoV-2 infection. In their cohort, $4.4 \%$ of the hospitalised patients and $5.3 \%$ of outpatients were daily smokers. Finally, they compared these results with the French general population (daily smokers' rate of $25.4 \%$ ). An increased susceptibility to SARS-COV2 infection in smokers was suggested. ${ }^{8}$

Since our study population is predominantly Spanish, we reviewed data regarding current smokers in Spain. These data have been reported by the Instituto Nacional de Estadística in 2017. The survey involved 29195 individuals interviewed between October 2016 and October 2017. They were classified in daily smokers, occasional smokers, former smokers and non-smokers. Smoking prevalence in 2017 among men was approximately $25.6 \%$ and $18.8 \%$ in women. It is worth pointing out that these rates are notably higher than the smoking rates recorded in our Spanish cohort (5\%). Although this imbalance draws attention, it should be considered that smoking status has been assessed only in hospitalised patients with COVID-19, thus symptomatic individuals fulfilling admission criteria. Since most COVID-19 studies have been performed in symptomatic patients, the smokers' lower susceptibility to have COVID-19 infection cannot be extrapolated from these data. Moreover, considering ex-smokers, the prevalence of current and former smokers reaches $21 \%$ in our cohort. This percentage approaches those observed in the general population.

Conversely, it has been suggested that ACE2 is upregulated in the airway epithelium of smokers. ${ }^{23}$ As previously stated, increased levels of ACE2 gene expression have been reported in samples taken from smokers in comparison with non-smokers ${ }^{28}$; thus, smokers may be more susceptible to SARS-CoV-2 infection. Similarly, in a study in resected lung specimens, Leung et alfound an increased rate of ACE2 gene expression in smokers. ${ }^{29}$
It remains to be seen if smokers are more prone to contracting SARS-CoV-2. Despite its relevance, the current data do not answer this question. It is also worth noting that the true prevalence of COVID-19 infection rates in the general population is likely underestimated.

\section{Limitations}

In our study, only hospitalised patients with COVID-19 were evaluated; therefore, it is clear that these patients had a more severe clinical course.

Moreover, as this study is an observational study, there is the potential for bias, given the nature of the study design. It must be considered that many individuals may be asymptomatic, and it is not currently possible to establish the real prevalence of smoking among all COVID-19 cases.

Furthermore, another limitation of our study was the fact that the number of pack-years of smoking was not recorded in our database and, therefore, it was not possible to classify the patients following this interesting criterion. Likewise, the number smoke-free years was not available, which would have provided a more accurate classification of previous risk in former smokers.

\section{CONCLUSIONS}

In conclusion, current smoking has a detrimental impact on COVID-19 prognosis. A history of active smoking is related to worse COVID-19 outcomes, with increased risk of mortality and the combined event, after adjusting for comorbidities. Likewise, a greater risk of mortality was still found among former smokers, compared with non-smokers.

\section{Author affiliations}

${ }^{1}$ Cardiology, Hospital Clinico Universitario San Carlos Instituto Cardiovascular, Madrid, Spain

${ }^{2}$ Cardiology/Internal Medicine, Hospital Clinico Universitario San Carlos Instituto Cardiovascular, Madrid, Spain

${ }^{3}$ Cardiology, University Hospital of Santiago de Compostela. Fundación IMAS, Galicia, Spain

${ }^{4}$ Cardiology, Hospital General Universitario de Guadalajara, Guadalajara, Spain ${ }^{5}$ Cardiology, Hospital La Paz, Madrid, Spain

${ }^{6}$ Cardiology, Hospital Clínico Universitario de Valladolid, Valladolid, Spain ${ }^{7}$ Cardiology/Emergency department, Hospital Universitario de Getafe, Madrid, Spain

${ }^{8}$ Cardiology, Hospital Universitario Clinica Puerta de Hierro, Madrid, Spain

${ }^{9}$ Cardiology, Hospital Severo Ochoa, Madrid, Spain

${ }^{10}$ Cardiology, Hospital Universitario Virgen de la Victoria; IBIMA. CIBERCV, Malaga, Spain

${ }^{11}$ Cardiology, Policlinico di Bari Ospedale Giovanni XXIII, Bari, Italy

${ }^{12}$ Cardiology, Azienda Ospedaliero Universitaria San Luigi Gonzaga, Orbassano, Italy

${ }^{13}$ Cardiology, Hospital Alvaro Cunqueiro, Vigo, Spain

${ }^{14}$ Cardiology, Hospital Infanta Sofia, Madrid, Spain

${ }^{15}$ Cardiology, Hospital General del Norte de Guayaquil, Guayaquil, Ecuador

${ }^{16}$ Emergency department, Hospital Universitario Principe de Asturias, Madrid, Spain

${ }^{17}$ Cardiology, Instituto de Cardiología y Cirugía Cardiovascular, La Habana, Cuba

${ }^{18}$ Cardiology, Sant'Andrea Hospital, Rome, Italy

${ }^{19}$ Cardiology, Hospital de Manises, Manises, Spain

${ }^{20}$ Cardiology, Hospital Nuestra Señora de América, Madrid, Spain

${ }^{21}$ Cardiology, University Hospital Heidelberg, Heidelberg, Germany

${ }^{22}$ The Second Affiliated Hospital of Southern University of Science and Technology,

Shenzhen, China

${ }^{23}$ Cardiology, Hospital de Especialidades Fuerzas Armadas N 1, Quito, Ecuador 
${ }^{24}$ Pneumology, Hospital Clínico Universitario, Incliva. Universidad de Valencia, Valencia, Spain

Acknowledgements I would like to express my appreciation to Dr Breda Hennessey for her careful reading of the manuscript and thoughtful advice.

Collaborators HOPE-COVID-19 (Health Outcome Predictive Evaluation for COVID-19, NCT04334291) investigators.

Contributors All authors contributed to the data collection, critical revision of the article and final approval of the version to be published. Conception or design of the work and data analysis and interpretation: IN-G and CF. Drafting the article: CE-P and IN-G. Patients were not involved in the design of the study.

Funding The authors have not declared a specific grant for this research from any funding agency in the public, commercial or not-for-profit sectors.

Competing interests None declared.

Patient consent for publication Not required.

Ethics approval This study has been conducted in accordance with the Declaration of Helsinki principles and Good Clinical Practice Guidelines. It has been approved by ethics research committee from the Hospital Clínico San Carlos (Madrid, Spain) (20/241-E) and the Spanish Agency for Medicines and Health Products classification (EPA-OD). Due to the pandemic situation, it was not appropriate to involve patients or the public in the design of our research.

Provenance and peer review Not commissioned; externally peer reviewed by Sridhar Chilimuri, USA.

Data availability statement Data are available upon reasonable request (Health Outcome Predictive Evaluation for COVID-19 Registry)

Supplemental material This content has been supplied by the author(s). It has not been vetted by BMJ Publishing Group Limited (BMJ) and may not have been peer-reviewed. Any opinions or recommendations discussed are solely those of the author(s) and are not endorsed by BMJ. BMJ disclaims all liability and responsibility arising from any reliance placed on the content. Where the content includes any translated material, BMJ does not warrant the accuracy and reliability of the translations (including but not limited to local regulations, clinical guidelines, terminology, drug names and drug dosages), and is not responsible for any error and/or omissions arising from translation and adaptation or otherwise.

Open access This is an open access article distributed in accordance with the Creative Commons Attribution Non Commercial (CC BY-NC 4.0) license, which permits others to distribute, remix, adapt, build upon this work non-commercially, and license their derivative works on different terms, provided the original work is properly cited, appropriate credit is given, any changes made indicated, and the use is non-commercial. See: http://creativecommons.org/licenses/by-nc/4.0/.

\section{ORCID iD}

Carolina Espejo-Paeres http://orcid.org/0000-0002-7196-9260

\section{REFERENCES}

1 WHO Director-General's opening remarks at the media briefing on COVID-19 - 11 March 2020. Available: https://www.who.int

2 Li Y-C, Bai W-Z, Hashikawa T. The neuroinvasive potential of SARSCoV2 may play a role in the respiratory failure of COVID-19 patients. $J$ Med Virol 2020;92:552-5.

3 Cao Y, Liu X, Xiong L, et al. Imaging and clinical features of patients with 2019 novel coronavirus SARS-CoV-2: a systematic review and meta-analysis. J Med Virol 2020;92:1449-59.

4 Guan W-J, Ni Z-Y, Hu Y, et al. Clinical characteristics of coronavirus disease 2019 in China. N Engl J Med 2020;382:1708-20.

5 Zhao Q, Meng M, Kumar R, et al. The impact of COPD and smoking history on the severity of COVID-19: a systemic review and metaanalysis. J Med Virol 2020;92:1915-21.

6 Liu W, Tao Z-W, Wang L, et al. Analysis of factors associated with disease outcomes in hospitalized patients with 2019 nove coronavirus disease. Chin Med J 2020;133:1032-8.

7 Lippi G, Henry BM. Active smoking is not associated with severity of coronavirus disease 2019 (COVID-19). Eur J Intern Med 2020;75:107-8.

8 Miyara M, Tubach F, Martinez V. Low rate of daily smokers in patients with symptomatic COVID-19. medRxiv 2020.
9 Huang C, Wang Y, Li X, et al. Clinical features of patients infected with 2019 novel coronavirus in Wuhan, China. The Lancet 2020;395:497-506.

10 Yang X, Yu Y, Xu J, et al. Clinical course and outcomes of critically ill patients with SARS-CoV-2 pneumonia in Wuhan, China: a singlecentered, retrospective, observational study. Lancet Respir Med 2020;8:475-81

11 Zhang J-J, Dong X, Cao Y-Y, et al. Clinical characteristics of 140 patients infected with SARS-CoV-2 in Wuhan, China. Allergy 2020;75:1730-41.

12 Mo P, Xing Y, Xiao Y, et al. Clinical characteristics of refractory COVID-19 pneumonia in Wuhan, China. Clin Infect Dis 2020;270.

13 Wan S, Xiang Y, Fang W, et al. Clinical features and treatment of COVID-19 patients in northeast Chongqing. J Med Virol 2020;92:797-806.

14 Liu J, Ouyang L, Guo P. Epidemiological, clinical characteristics and outcome of medical staff infected with COVID-19 in Wuhan, China: a retrospective case series analysis. medRxiv 2020.

15 Signes-Costa J, Núñez-Gil IJ, Soriano JB, et al. Prevalence and 30-day mortality in hospitalized patients with Covid-19 and prior lung diseases. Archivos de Bronconeumología 2021;57:13-20.

16 Nan Y, Xi Z, Yang Y. The 2015 China adult tobacco survey: exposure to second-hand smoke among adults aged 15 and above and their support to policy on banning smoking in public places 2016;37:810-5.

17 Garufi G, Carbognin L, Orlandi A, et al. Smoking habit and hospitalization for severe acute respiratory syndrome coronavirus 2 (SARS-CoV-2)-related pneumonia: The unsolved paradox behind the evidence. Eur J Intern Med 2020;77:121-2.

18 Wong CK, Lam CWK, Wu AKL, et al. Plasma inflammatory cytokines and chemokines in severe acute respiratory syndrome. Clin Exp Immunol 2004;136:95-103.

19 Ruan Q, Yang K, Wang W, et al. Clinical predictors of mortality due to COVID-19 based on an analysis of data of 150 patients from Wuhan, China. Intensive Care Med 2020;46:846-8.

20 Chen N, Zhou M, Dong X, et al. Epidemiological and clinical characteristics of 99 cases of 2019 novel coronavirus pneumonia in Wuhan, China: a descriptive study. The Lancet 2020;395:507-13.

21 Qiu F, Liang C-L, Liu H, et al. Impacts of cigarette smoking on immune responsiveness: up and Down or upside down? Oncotarget 2017;8:268-84

22 Shiels MS, Katki HA, Freedman ND, et al. Cigarette smoking and variations in systemic immune and inflammation markers. J Natl Cancer Inst 2014;106.

23 Brake SJ, Barnsley K, Lu W, et al. Smoking upregulates angiotensinconverting enzyme-2 receptor: a potential adhesion site for novel coronavirus SARS-CoV-2 (Covid-19). J Clin Med 2020;9:841.

24 Hamming I, Timens W, Bulthuis MLC, et al. Tissue distribution of ACE2 protein, the functional receptor for SARS coronavirus. A first step in understanding SARS pathogenesis. J Pathol 2004;203:631-7.

25 Wrapp D, Wang N, Corbett KS, et al. Cryo-Em structure of the 2019nCoV spike in the prefusion conformation. Science 2020;367:1260-3.

26 Wan Y, Shang J, Graham R, et al. Receptor recognition by the novel coronavirus from Wuhan: an analysis based on decade-long structural studies of SARS coronavirus. J Virol 2020;94.

27 Oakes JM, Fuchs RM, Gardner JD, et al. Nicotine and the reninangiotensin system. Am J Physiol Regul Integr Comp Physiol 2018;315:R895-906.

28 Cai G. Tobacco-Use disparity in gene expression of ACE2. the Receptor of 2019-nCov 2020 https://www.preprints.org

29 Leung JM, Yang CX, Tam A, et al. ACE-2 expression in the small airway epithelia of smokers and COPD patients: implications for COVID-19. Eur Respir J 2020;55. doi:10.1183/13993003.006882020. [Epub ahead of print: 14 May 2020].

30 Alraddadi BM, Watson JT, Almarashi A, et al. Risk factors for primary middle East respiratory syndrome coronavirus illness in humans, Saudi Arabia, 2014. Emerg Infect Dis 2016;22:49-55.

31 Patanavanich R, Glantz SA. Smoking is associated with COVID-19 progression: a meta-analysis. Nicotine Tob Res 2020;22:1653-6.

32 Jiménez-Ruiz CA, López-Padilla D, Alonso-Arroyo A. COVID-19 and smoking: a systematic review and meta-analysis of the evidence. Arch Bronconeumol 2021;57:21-34.

33 Lowe KE, Zein J, Hatipoglu U, et al. Association of smoking and cumulative Pack-Year exposure with COVID-19 outcomes in the Cleveland clinic COVID-19 registry. JAMA Intern Med 2021;181:709-11.

34 Vardavas CI, Nikitara K. COVID-19 and smoking: a systematic review of the evidence. Tob Induc Dis 2020;18:20.

35 Adrish M, Chilimuri S, Mantri N, et al. Association of smoking status with outcomes in hospitalised patients with COVID-19. BMJ Open Respir Res 2020;7:e000716. 